\title{
Article
}

\section{Online Real-Time Monitoring System through Using Adaptive Angular-Velocity VKF Order Tracking}

\author{
Ting-Chi Yeh", Min-Chun Pan ${ }^{1, *}$ and Duc Do Le ${ }^{2}$ \\ ${ }^{1}$ Department of Mechanical Engineering, National Central University, Jhongli 320, Taiwan \\ ${ }^{2}$ Department of Machine Tools and Tribology, Hanoi University of Science and Technology, Vietnam \\ * Correspondence: pan_minc@cc.ncu.edu.tw; Tel.: +886-3-4267312; Fax: +886-3-4254501.
}

\begin{abstract}
When a rotary machine is running, from which the acquired vibro-acoustic signals enable to reveal its operation status and health condition. The study proposed a DSP-based adaptive angular-velocity Vold-Kalman filtering order tracking (AV2KF_OT) algorithm with an online realtime nature for signal interpretation and machine condition monitoring. Theoretical derivation and numerical implementation of computation schemes are briefly introduced. An online real-time monitoring system based on the AV2KF_OT algorithm, which was implemented through both a digital signal processor and a user interface coded by using LabVIEW®, was developed. Two experimental tasks were applied to justify the proposed technique, including (i) the detection of startup on the fluid-induced whirl performed through a journal-bearing rotor rig, and (ii) the separation of close orders from the measured signals of a multifunction transmission-element ballbearing bench.
\end{abstract}

Keywords: Rotary machinery; Adaptive order tracking; Online real-time monitoring.

\section{Introduction}

Various mechanical systems have their specific ways to transmit power for motion. Rotary machine elements such as gear sets, belt drives, or chain modules play an important role in the mechanical systems. Lots of researches have endeavored to diagnose and control the state of the machine health, as a result to avoid or solve the machine problems and save the cost. Mechanical systems under periodic loading due to rotary operation usually respond in measurements with a superposition of sinusoids whose frequencies are integer (or fractional integer) multiples of the reference shaft speed, which is usually the revolution speed of the loading with the slowest one. The fundamental frequency corresponding to the shaft speed is called a basic order. The order tracking (OT) is one of effective tools for the signal interpretation and diagnosis of rotary machinery. This technique enables to extract the sinusoidal contents of measurement from a mechanical system under periodic loading, and is frequently applied in analyzing dynamic signals measured from rotary machines.

All developed OT techniques can be classified into two categories including waveform nonreconstruction and reconstruction schemes [1]. The waveform non-reconstruction schemes, which can process signals to be characterized into a rpm-frequency or rpm-order plane but cannot reconstruct the time waveforms of specific orders, include the short-time Fourier transform (STFT) based order analysis, and the resampling scheme [2-4]. In contrast, the waveform reconstruction schemes, which enable to extract specific spectral/order components and reconstruct their time waveform, include Vold-Kalman filtering OT (VKF_OT) techniques [5-9], the recursive least-squares (RLS) OT [10,11], and Gabor OT (GOT) [1,3,12,13], etc.

The conventional OT approaches like the windowed Fourier transform (WFT) and the resampling methods primarily based on Fourier analysis have limited resolution in some situations and suffer from a number of shortcomings such as not available to obtain the waveform of an individual order of interest. Vold et al. [5-7] proposed a Kalman filtering based computation scheme for the estimation of high resolution, slew rate independent order components. Pan and Lin $[8,9]$ 
further explored the theoretical details of VKF_OT and compared the differences of these two VKF_OT algorithms, including the angular-displacement and angular-velocity VKF_OTs. But it is noted that these two VKF_OT schemes must be computed off-line and implemented as postprocessing techniques; i.e., they were not real-time applications yet. The computations for both the angular-velocity and angular-displacement VKF_OTs are rather time-consuming since the whole acquired signal to be evaluated for specific order/spectral components is contained in the huge matrix manipulation [8]. To cope with this problem, Haykin et al. [14] exploit a state-pace model using the extended RLS algorithm to deal with the tracking of a chirped sinusoid in additive noise, analogous to the OT for rotary machinery. Bai et al. [10] proposed an adaptive OT technique based on the RLS algorithm to track frequency-varying bandpass signals. The one-step predictor was employed in the Kalman filtering computation in Wu's et al. study [11]. The adaptive real-time processing VKF_OT algorithms incorporating the angular-displacement and angular-velocity structural equations, respectively, were also derived and realized in our previous studies [15, 16]. Pan and Wu [17] proposed and implemented an adaptive VKF_OT approach based upon adapted one-step prediction scheme for condition monitoring of rotating machine. The computation scheme makes real-time processing feasible and overcomes the drawbacks of the original VKF_OT scheme [8]. Besides, Gabor OT $[1,12]$ and some hybrid approaches [18-21] for multiple order extraction were also proposed and realized in past decades; especially, it is rather straightforward to use the GOT technique that associates with flexible tracking-mask selection to extract and reconstruct spectral components. The information of shaft speed can even be missing while the GOT is applied for rotary machines. The GOT technique was proposed with extremely good selectivity on the extracted signal component [12]. Pan and Chiu [1] developed an improved algorithm of GOT to tackle the crossing-order/spectral problem and get rid of the precise measurement of shaft speed as well.

This paper is to present an online real-time monitoring system equipped with the AV2KF_OT scheme to real-time track order/spectral components and implemented with a digital signal processor (DSP) and a LabVIEWø coded user interface through which the adaptive processing algorithm was realized and implemented. Thus, the concurrent computation can be achieved as soon as measured data streams are acquired. Two sets of simulation signals for (i) mimicking various whirl conditions tuned through a center-line adjuster of a journal-bearing rotor test rig and (ii) imitating close-order vibration arising from multifunction transmission-element test rig were synthesized to validate the computation scheme. Afterwards, two case studies through journal-bearing rotor test bench and multifunction transmission-element test bench were investigated to justify the usefulness of the proposed method.

\section{Theoretical Background}

The basic idea of the conventional angular-velocity VKF_OT is to define local constraints, the socalled structural equation and data equation $[7,8]$, which make the unknown complex envelopes smooth and relate the tracked orders to the measured signal. These two equations are solved by using a least-squares technique through a large number of matrix computations. It resembles the Wiener filter in mathematical operation. Furthermore, the Kalman filter is essentially a recursive algorithm whose solution converges to the optimum Wiener solution in some statistical sense. The mathematical model of the Kalman filter can be embodied in a pair of equations, i.e., the process and measurement equation [15], as follows.

Process equation: $\quad \mathbf{x}(n+1)=\mathbf{F}(n+1, n) \mathbf{x}(n)+\mathbf{v}_{1}(n)$

Measurement equation: $\quad \mathbf{y}(n)=\mathbf{C}(n) \mathbf{x}(n)+\mathbf{v}_{2}(n)$

The proposed adaptive angular-velocity VKF_OT provides us a recursive algorithm without having to evaluate a huge inverse matrix with all observed time points. More precisely, this adaptive OT scheme is to derive and rewrite the structural and data equation in a form of process and measurement equation, respectively. Thus the original (off-line) angular-velocity VKF_OT $[7,8]$ with 
its computational complexity in solving the inverse matrix can be dramatically improved by using Kalman filtering based on one step prediction scheme.

\subsection{Structural equation}

For a second-order homogeneous ordinary differential equation (ODE)

$$
\frac{d^{2} x(t)}{d t^{2}}+\omega^{2} x(t)=0
$$

its complementary solution is

$$
x(t)=K_{1} e^{j \omega t}+K_{2} e^{-j \omega t},
$$

where $K_{1}$ and $K_{2}$ are arbitrary constants. The discrete form of Eq. (4) can be presented as

$$
x(n \Delta T)=K_{1} e^{j \omega n \Delta T}+K_{2} e^{-j \omega n \Delta T},
$$

where $t=n \Delta T, n=1,2,3, \ldots$; and $\Delta T$ denotes sampling time spacing. Assume $d_{1}=e^{j w \Delta T}$ and $d_{2}=e^{-j w \Delta T}$ respectively, then Eq. (5) becomes

$$
x(n)=K_{1}\left(d_{1}\right)^{n}+K_{2}\left(d_{2}\right)^{n} .
$$

Thus, the characteristic equation of (6) can be expressed as

$$
H(D)=\left(D-d_{1}\right)\left(D-d_{2}\right),
$$

where the operator notation $D$ denotes a discrete-time delay, e.g. $D x(n)=x(n-1)$. The discrete time signal $x(n)$ satisfies a second-order difference equation $H(D) x(n)=0$, i.e.

$$
x(n)-2 \cos (\omega \Delta T) x(n-1)+x(n-2)=0,
$$

where $x(n)$ is the order component with an angular frequency $\omega$ in $\mathrm{rad} / \mathrm{s}$ to be computed. In realworld applications, an order component of interest with changing frequency to be tracked is usually contaminated with noise and other sinusoids. A heterogeneous term, $s(n)$, can be introduced to allow the sinusoidal wave to change its amplitude, phase and frequency slightly. Therefore, Eq. (8) can be modified as

$$
x(n)-2 \cos (\omega \Delta T) x(n-1)+x(n-2)=\varepsilon(n) .
$$

Equation (9) is the structural equation of the angular-velocity VKF_OT for independently a single order to be tracked. Likewise, to extract $K$ different spectral/order components simultaneously, the structural equation of each component can be rewritten as

$$
x_{k}(n)-2 \cos \left(\omega_{k}(n) \Delta T\right) x_{k}(n-1)+x_{k}(n-2)=\varepsilon_{k}(n),
$$


where $k$ represents the order/spectral index to be computed. Equation (10) can be further shown in matrix form like

$$
\left[\begin{array}{l}
x_{k}(n) \\
x_{k}(n+1)
\end{array}\right]=\left[\begin{array}{cc}
0 & 1 \\
-1 & 2 \cos \left(\omega_{k}(n) \Delta T\right)
\end{array}\right]\left[\begin{array}{l}
x_{k}(n-1) \\
x_{k}(n)
\end{array}\right]+\left[\begin{array}{l}
0 \\
\varepsilon_{k}(n)
\end{array}\right]
$$

Let $\underline{\underline{x}}_{k}(n+1)=\left[\begin{array}{l}x_{k}(n) \\ x_{k}(n+1)\end{array}\right], \underline{x}_{k}(n)=\left[\begin{array}{l}x_{k}(n-1) \\ x_{k}(n)\end{array}\right], \mathbf{M}_{k}(n)=\left[\begin{array}{cc}0 & 1 \\ -1 & 2 \cos \left(\omega_{k} \Delta T\right)\end{array}\right]$, and $\underline{\varepsilon}_{k}(n)=\left[\begin{array}{l}0 \\ \varepsilon_{k}(n)\end{array}\right]$, then (11) can be expressed as

$$
\underline{x}_{k}(n+1)=\mathbf{M}_{k}(n) \underline{x}_{k}(n)+\underline{\varepsilon}_{k}(n) .
$$

To simultaneously track multiple order/spectral components, Eq. (12) can be augmented to all $\mathrm{K}$ order/spectral components to be extracted as following.

$$
\left[\begin{array}{c}
\underline{x}_{1}(n+1) \\
\underline{x}_{2}(n+1) \\
\vdots \\
\underline{x}_{K}(n+1)
\end{array}\right]=\left[\begin{array}{ccccccc}
\mathbf{M}_{1}(n) & \mathbf{0} & \mathbf{0} & \mathbf{0} & \cdots & \mathbf{0} & \mathbf{0} \\
\mathbf{0} & \mathbf{M}_{2}(n) & \mathbf{0} & \mathbf{0} & \cdots & \mathbf{0} & \mathbf{0} \\
\mathbf{0} & \mathbf{0} & \mathbf{M}_{3}(n) & \mathbf{0} & \cdots & \mathbf{0} & \mathbf{0} \\
\vdots & \vdots & \vdots & \vdots & \ddots & \vdots & \vdots \\
\mathbf{0} & \mathbf{0} & \mathbf{0} & \mathbf{0} & \cdots & \mathbf{0} & \mathbf{M}_{k}(n)
\end{array}\right]\left[\begin{array}{c}
\underline{x}_{1}(n) \\
\underline{x}_{2}(n) \\
\vdots \\
\underline{x}_{K}(n)
\end{array}\right]+\left[\begin{array}{c}
\underline{\varepsilon}_{1}(n) \\
\underline{\varepsilon}_{2}(n) \\
\vdots \\
\underline{\varepsilon}_{K}(n)
\end{array}\right] .
$$

Similarly, the symbolized form of (13) becomes

$$
\mathbf{X}(n+1)=\mathbf{F}(n+1, n) \mathbf{X}(n)+\mathbf{E}(n) .
$$

Equation (14) is actually the structural equation of the adaptive angular-velocity VKF order tracking, and it satisfies the algebraic formality of process equation, Eq. (1), for Kalman filtering.

\subsection{Data Equation}

Assuming a dynamic signal $y(n)$, measured from the operation of a rotary machine, contains a target order $x_{k}(n)$ that satisfies the structural equation, as well as other spectral/order components and embedded noise, it possesses a formality of

$$
y(n)=x_{k}(n)+\eta(n),
$$

where the component $\eta(n)$ is composed of other not-concerned spectral/order components and measurement artifacts. Equation (15) is the data equation of the angular-velocity VKF_OT for independently tracking a single order or spectral component. For simultaneously tracking multiple rotation-related orders and/or spectral components such as resonances, the measured signal $y(n)$ can be rewritten as a combination of several order/spectral components, $x_{k}(n)$, and measurement artifacts $\eta(n)$, i.e.,

$$
y(n)=\sum_{k=1}^{K} x_{k}(n)+\eta(n)
$$


where $K$ denotes the number of order/spectral components to be tracked. Moreover, Eq. (16) can be expressed with a state vector, $\boldsymbol{X}(n)$,

$$
y(n)-\left[\begin{array}{lllll}
\underline{I}_{1}(n) & \underline{I}_{2}(n) & \underline{I}_{3}(n) & \cdots & \underline{I}_{K}(n)
\end{array}\right]\left[\begin{array}{c}
\underline{x}_{1}(n) \\
\underline{x}_{2}(n) \\
\vdots \\
\underline{x}_{K}(n)
\end{array}\right]=\eta(n)
$$

where $\underline{I}_{k}(n)=\left[\begin{array}{ll}0 & 1\end{array}\right], k=1,2, \ldots, K$. Let $\mathbf{I}(n)=\left[\begin{array}{lllll}\underline{I}_{1}(n) & \underline{I}_{2}(n) & \underline{I}_{3}(n) & \cdots & \underline{I}_{K}(n)\end{array}\right]$ and $\mathbf{X}(n)=\left[\begin{array}{c}\underline{x}_{1}(n) \\ \underline{x}_{2}(n) \\ \vdots \\ \underline{x}_{K}(n)\end{array}\right]$, then Eq. (17)

can be expressed as

$$
y(n)=\mathbf{I}(n) \mathbf{X}(n)+\eta(n) .
$$

Equation (18) is the data equation of the adaptive angular-velocity VKF_OT, and has the same form as the measurement equation, Eq. (2) of a Kalman filter.

\subsection{Waveform Evaluation Based on Kalman filtering}

As mentioned previously, the deduced structural Eq. (14), and data Eq. (18), are formulated identical to the process and measurement equations of Kalman filtering. Consequently, the ordertracking problem can be considered as a state estimation problem like the procedure of Kalman filtering. The tracked order waveforms can be recursively solved by using Kalman filtering based on one-step prediction as illustrated in [14,15].

In the process equation for Kalman filtering, the $2 \mathrm{~K} \times 1$ vector $\mathrm{Z}(\mathrm{n})$ represents process noise, modeled as a zero-mean, white-noise process whose correlation matrix is defined as below

$$
E\left[\mathbf{Z}(n) \mathbf{Z}^{H}(m)\right]= \begin{cases}\mathbf{Q}_{1}(n), & n=m \\ 0, & n \neq m\end{cases}
$$

Accordingly, the correlation matrix of the process noise can be defined as

$$
E\left[\psi_{k}(n) \psi_{l}^{*}(m)\right]= \begin{cases}\mathbf{Q}_{1}^{k, l}(n), & n=m \cap k=l \\ 0, & n \neq m \cup k \neq l\end{cases}
$$

where $\psi \mathrm{k}(\mathrm{n})$ or $\psi \mathrm{l}(\mathrm{m})$ is the element in the process noise vector $Z(\mathrm{n}), \mathrm{k}, 1 \in \mathrm{j}(=1,2,3, \ldots$, and/or $K)$ represents the index of the tracked order components, $\mathbf{Q}_{1}^{k, l}(n)$ is the element at the $k^{\text {th }}$ row and the $l^{\text {th }}$ column of $\mathrm{Q}_{1}(\mathrm{n})$, and the asterisk denotes complex conjugation.

Likewise, the variable $\xi(n)$ in the measurement equation for Kalman filtering is the measurement noise, modeled as a zero-mean and white-noise process, whose correlation is defined by 


$$
E\left[\xi(n) \xi^{*}(m)\right]= \begin{cases}Q_{2}(n), & n=m \\ 0, & n \neq m\end{cases}
$$

It is noted that the process noise $\psi_{k}(n)$ regards the higher order terms of the envelope $\left(a_{k}(n)\right)$ of each order component $x_{k}(n)$, and the measurement noise $\xi(n)$ relates to artifacts other than the order components of interest. It is assumed the process and the measurement noise are statistically independent, thus

$$
E\left[\psi_{k}(n) \xi^{*}(m)\right]=0 \text {, for all } n \text { and } m
$$

In order to design parameters $\mathrm{Q}_{1}(\mathrm{n})$ and $\mathrm{Q}_{2}(\mathrm{n})$, the weighting factor $\mathrm{r}(\mathrm{n})$, introduced in the angular-velocity VKF_OT [5], is employed into the error tolerance of both the structural and data equations, and defined as

$$
r(n) \equiv S_{\eta}(n) / S_{\varepsilon}(n)
$$

where $S_{\eta}(n)$ and $S_{\varepsilon}(n)$ are the standard deviations of measurement artifacts $\eta(n)$ and heterogeneous term $\varepsilon(n)$, respectively. Two standard deviations, $S_{\eta}(n)$ and $S_{\varepsilon}(n)$, are fulfilled Eq. (23). Correspondingly, the introduced weighting factor r(n) also satisfies Eq. (22) to be defined as Eq. (20) for all $n \neq m$. Therefore, $r(n)$ can be introduced into the computation of Kalman filtering for the adaptive AV VKF_OT scheme; further, the relationship among $r(n), Q_{1}(n)$ and $Q_{2}(n)$ is acquired as

$$
\frac{S_{\eta}^{2}(n)}{S_{\varepsilon}^{2}(n)}=r^{2}(n)=\frac{Q_{2}(n)}{\mathbf{Q}_{1}^{k, l}(n)},=>Q_{2}(n)=r^{2}(n) \mathbf{Q}_{1}^{k, l}(n)
$$

in which $\mathrm{Q}_{1}(\mathrm{n})$ is generally an identity matrix $\mathrm{I}_{2 \mathrm{~K} \times 2 \mathrm{~K}}$ multiplied by $\mathrm{q}_{1}$. The assignment of $\mathrm{Q}_{1}(\mathrm{n})$ can be set variously according to the nature of order or spectral components to be tracked, and $\mathrm{Q}_{2}(\mathrm{n})$ is a scalar obtained by the squared waiting factor multiplied by the index of $\mathrm{Q}_{1}(\mathrm{n})$.

As a result, the tracked order/spectral components, $X(n)$, can be recursively solved by using Kalman filtering based on one-step prediction [14], which accommodates the weighting factor, $r(n)$, and parameters of the proposed adaptive OT here for each time instant during data acquiring. In Eq. (11), the instantaneous frequency of a non-stationary order component, $\underline{x}_{k}(n)$, directly relates to the varying reference revolution speed. The tracked order component $\underline{x}_{k}(n)$ with known instantaneous frequency can hence be evaluated. The reference shaft speed in the unit of angular velocity needs to be measured for estimating the instantaneous frequency of a higher (multiple or fractional) interesting order.

\section{Experimental Setup and Real-Time Monitoring System}


Two rotary-system test bench were built up for the experimental work to justify the feasibility of the proposed scheme. Figure 1 shows a rotor test rig with a journal bearing. The test bench driven by a 90 -watt AC induction motor includes a shaft with $550 \mathrm{~mm}$ long and a diameter of $9.52 \mathrm{~mm}$, and a fluid-film bearing made of acrylics, filled with SAE 10 oil dyed in blue that allows visual inspection on the condition of the fluid film during experiments. Additionally, the bench was equipped by using a variable speed controller, two eddy current transducers to pick up vibration displacement of shaft, a centerline adjuster to regulate the journal position, and a speed transducer is installed between the motor and a flexible coupling to measure the rotor revolution speed.

A rotor dynamic system with a journal bearing may yield strong shaft shake due to lateral selfexcited rotor vibration, known as fluid-induced instability [22, 23]. Two phenomena, oil whirl and oil whip, occur at a relatively low and high shaft speed respectively, in which the whirl occurs when the journal operates close to the center of a bearing (low eccentricity). The revolution speed at which the instability onset occurs is called the threshold of instability. Oil whirl in general takes place in a range of 0.43 to 0.49 times of shaft revolution speed, while oil whip is usually associated with the first shaft bending mode and not influenced by the shaft speed occurs at a fixed frequency.

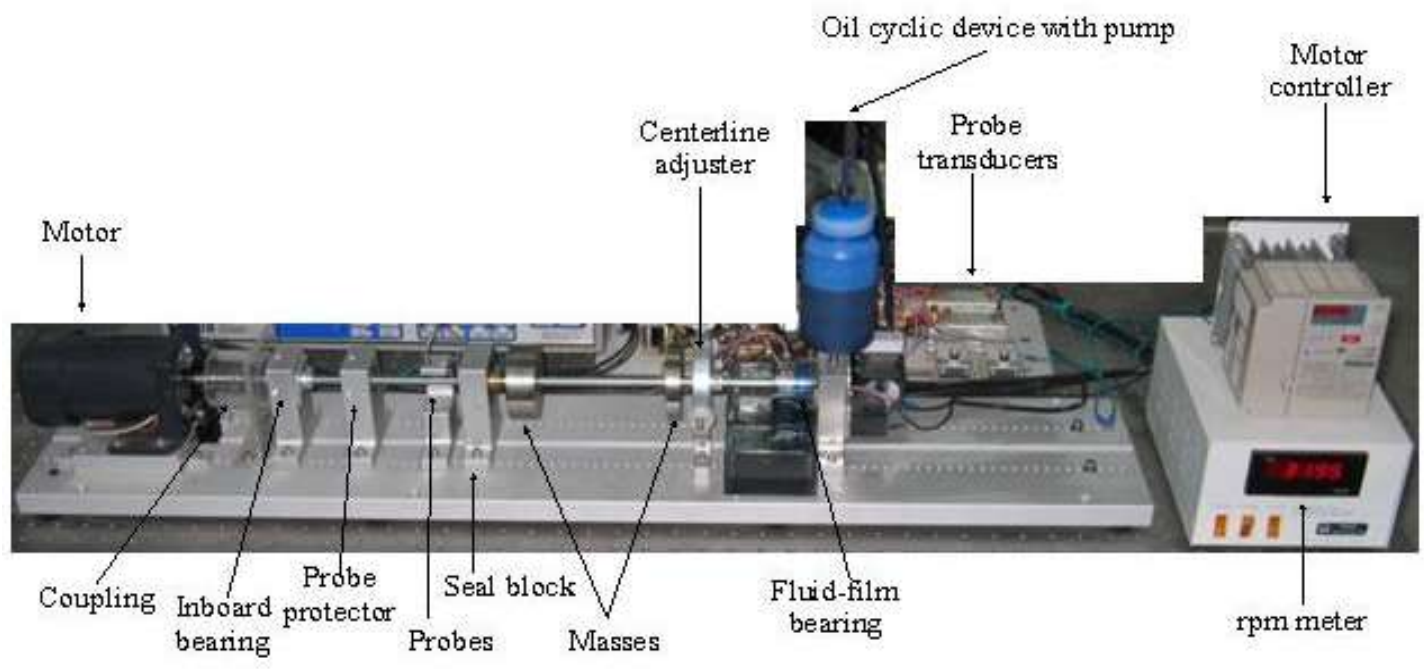

Fig. 1. Rotor test bench with journal bearing.

Besides, as shown in Fig. 2 a motor-driven multifunction transmission-element test bench was also constructed to investigate the characteristics of a transmission element system, whose machine parts include a servomotor as the power source, multiple transmission components including a two stage speed reduction gearbox with a speed ratio of 5 , an adjustable speed ratio belt driven set, and a roller chain set with a speed ratio of 12 to 7; further, an electromagnetic brake is employed to serve as a load of the system, and a two-piece flywheel set with radial screw holes is mounted on the main shaft for conducting necessary system balance.

To realize an online real-time monitoring system, a DSP (TI TMS320C6713) was employed as the computation kernel, together with two sets of I/O modules with a $125 \mathrm{kHz}$ sampling rate and eight channels for each. The adaptive angular-velocity VKF_OT scheme was coded through C++ and code composer (Sheldon Instruments). Besides, the graphical user interface was coded in an environment of LabVIEW for the parameter setting and handling of data acquisition, processing and illustration, as shown in Fig. 3.

The built-up monitoring system with using an adaptive velocity VKF_OT scheme was employed to monitor the status (whirl or whip) of journal-bearing rotor system and to separate close orders that arise from a multifunction transmission-element test bench. An appropriate response reacts as soon as the detection of incipient instability of motion through online real-time monitoring. This can prevent severe vibration from causing machine damage. During the DSP programming procedure, the most important thing is the memory configuration. Memory arrangement includes user interface input parameters, such as the sampling frequency $(f s)$, the length of the interception of data, and 
weighting factor, etc. Each obtained output signal is assigned to a different memory location. Once the memory position is allocated, the computer and the DSP card go through such a memory location corresponding to the read data. As shown in Fig. 4, the DSP programming that requires to read a specific memory location only needs to call the corresponding name by using the LabVIEW diagram of operating elements.

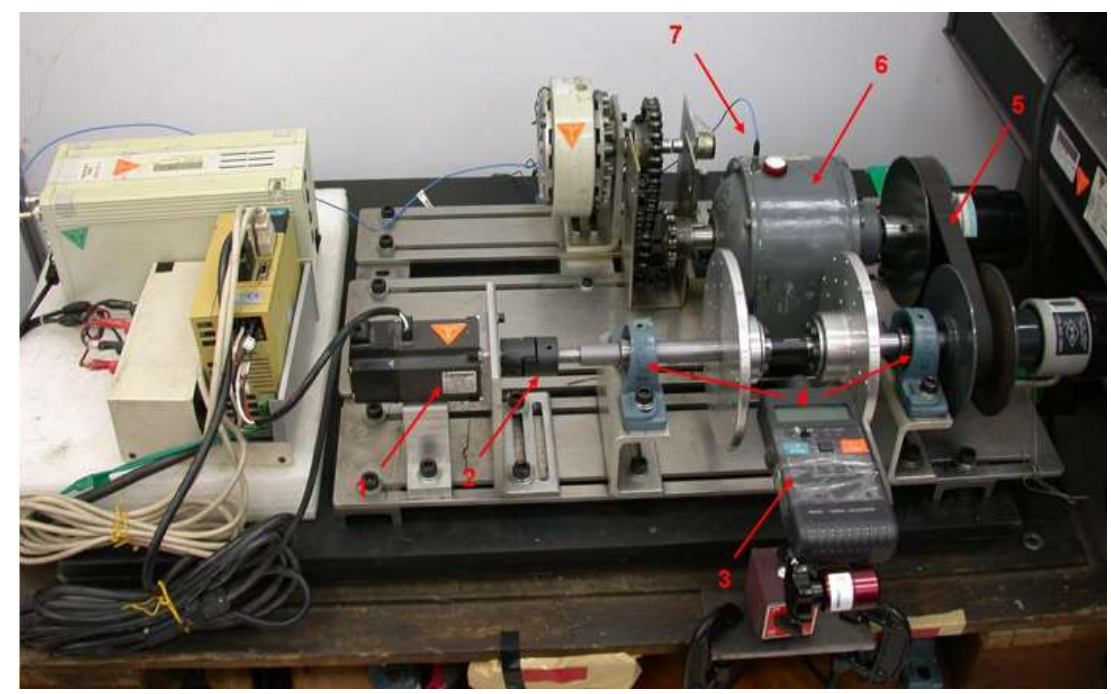

Fig. 2. Transmission-element test bench, where 1: AC servo motors, 2: flexible coupling, 3: tacogenerator, 4: pillow block ball bearings, 5: V-belt, 6: gearbox speed reducer, 7: accelerometer.

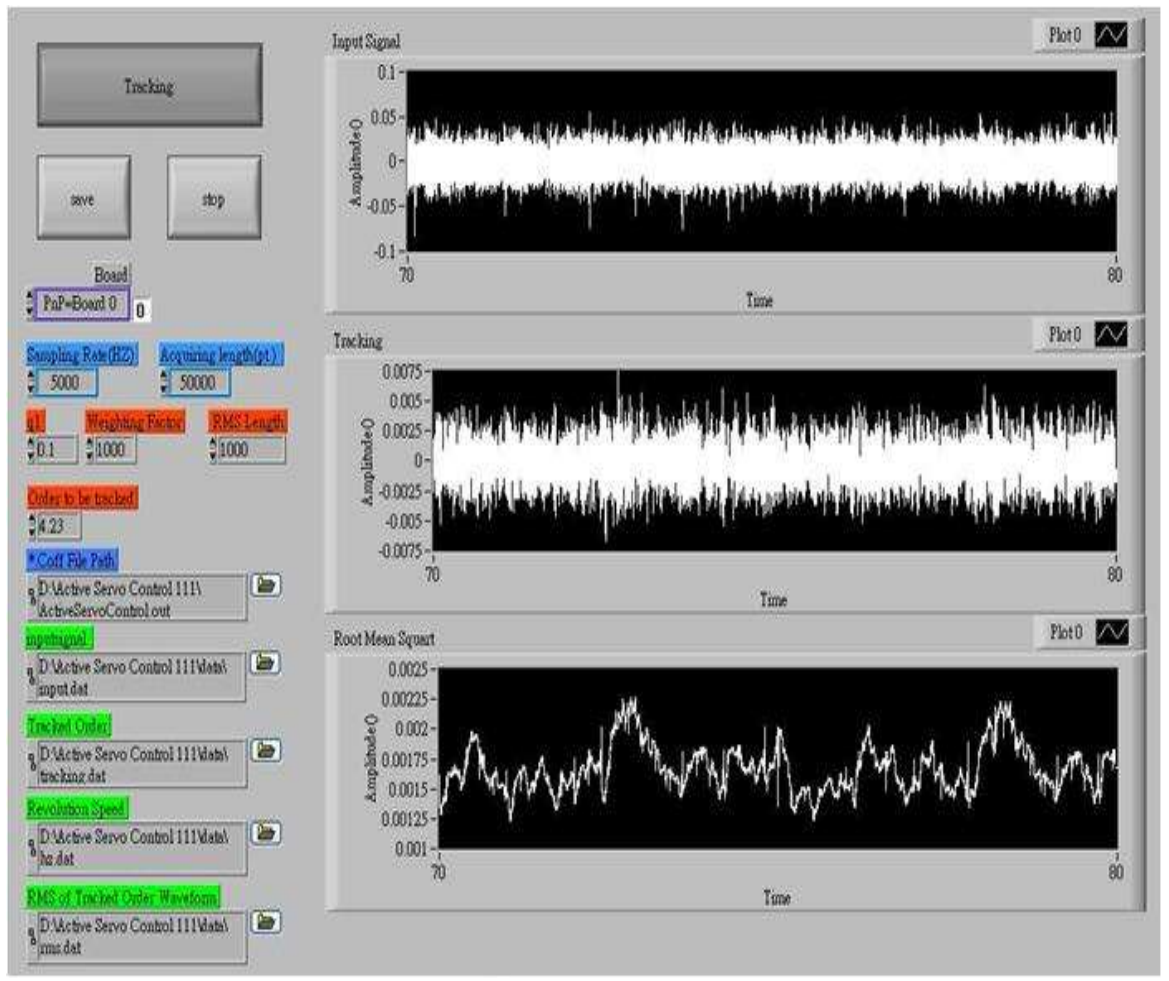

Fig. 3. Online real-time GUI using LabVIEW. 


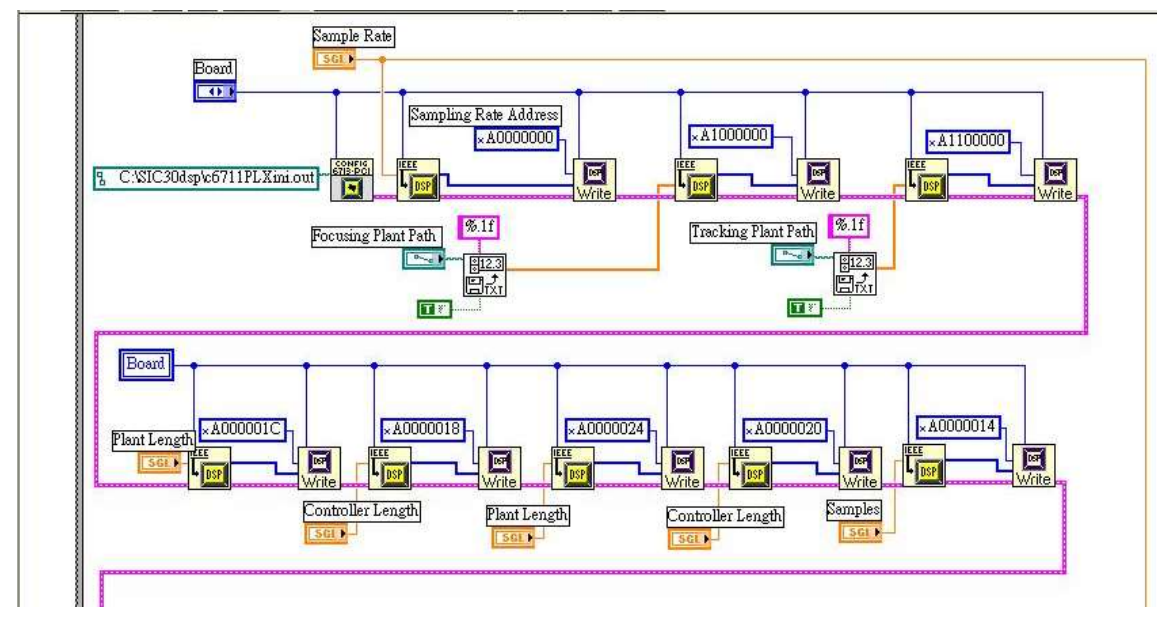

Fig. 4. LabVIEW diagram of operating elements.

\section{Simulation and Experimentation}

\subsection{Numerical Simulation}

Two synthesized signals are designated to justify the real-time monitoring feasibility of the proposed scheme; the one is for tracking the vibration response of whirl and whip phenomena and the other is for separating two close order vibration signatures along the run-up of shaft speed.

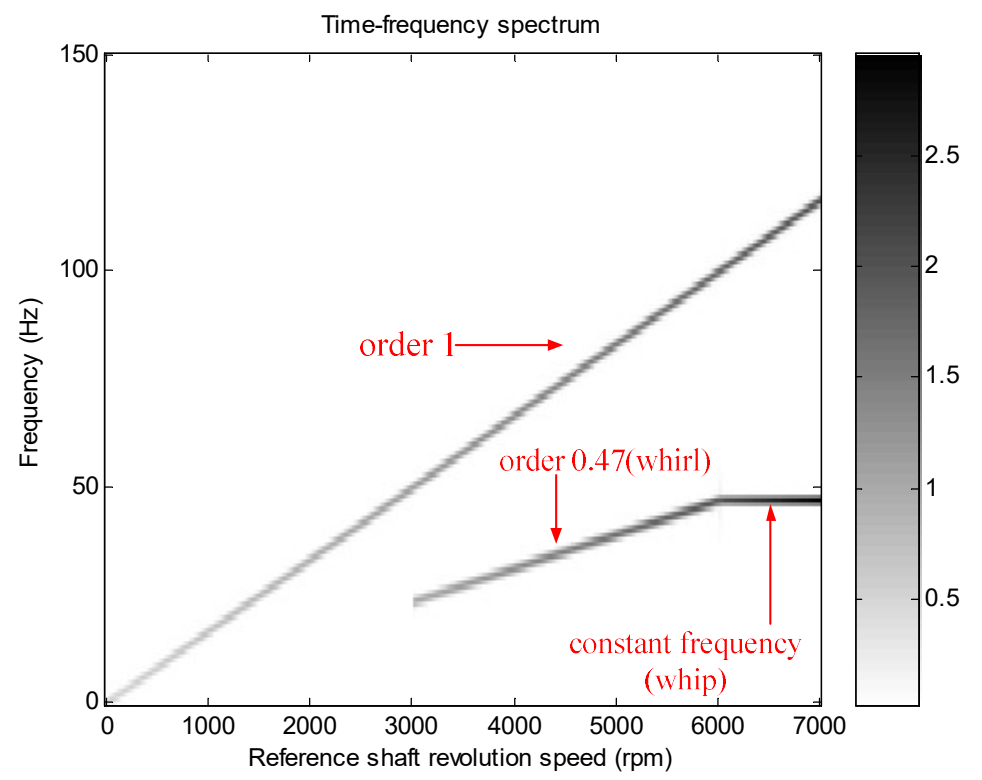

Fig. 5. rpm-spectrogram of synthetic signal 1 to characterize the occurrence of whirl.

\subsubsection{Synthetic signal 1: tracking of whirl and whip vibration responses}

The synthetic signal is composed of two order components, order 0.47 and order 1 corresponding to whirl response and unbalancing, respectively, and a fixed-frequency one to mimic whip signature. This imitates a journal-bearing test bench speeding up from stationary to $7000 \mathrm{rpm}$ in $180 \mathrm{~s}$ during the operation process. More precisely, the amplitude of order 1 increases from 1 to 4 constantly, order 0.47 starts from 2 at a speed of $3000 \mathrm{rpm}$ to 4 till $6000 \mathrm{rpm}$, and the $47-\mathrm{Hz}$ resonance (whip) occurs as the speed reaches $6000 \mathrm{rpm}$ and its amplitude increases from 4 to 6 till $7000 \mathrm{rpm}$. Figure 5 illustrates the rpm-spectrogram of the synthetic signal that characterizes the above described spectral/order 
components. The parameter setting for the computation of adaptive order tracking employs the weighting factor $r=2000, q_{1}=0.1$, and a sampling frequency $f_{s}=2000 \mathrm{~Hz}$; thus it yields the extracted waveform of order 0.47 (Fig. 6). It is found the order 0.47 component can be correctly extracted from 3000 to $6000 \mathrm{rpm}$ except an artifact signature is also extracted arising from order 1 component at the low speed. Therefore, the amplitude of order- 0.47 component, expressed as a root mean square (RMS) value, can be evaluated and employed for monitoring the occurrence of oil whirl.

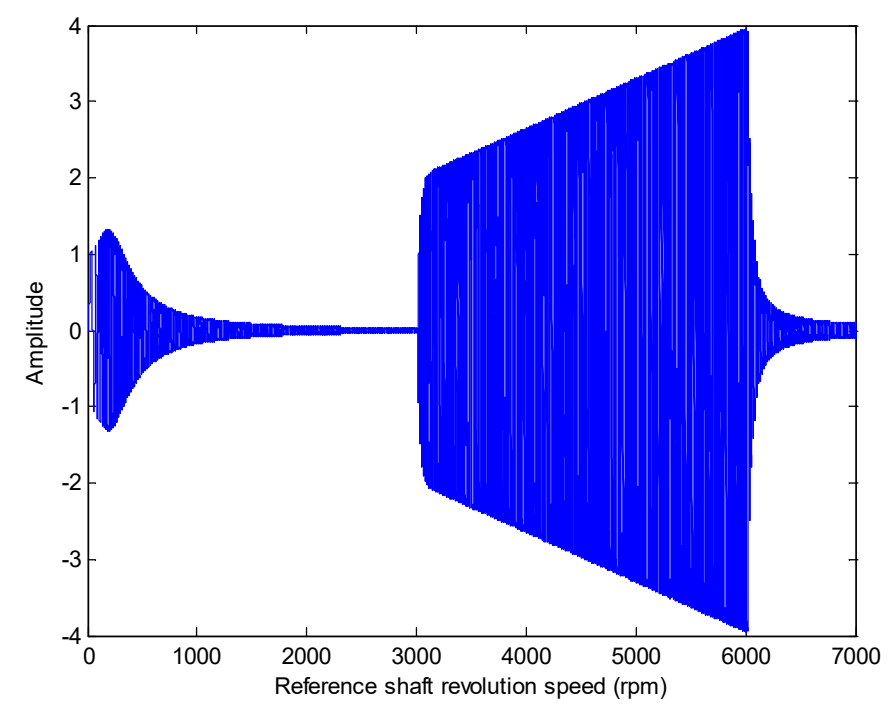

Fig. 6. Extracted order-0.47 waveform for synthetic signal 1.

\subsubsection{Synthetic signal 2: separation of close orders}

In this case, the test bench speeds up from stationary to $3000 \mathrm{rpm}$ in $5 \mathrm{~s}$, with the sampling frequency of $2000 \mathrm{~Hz}$. A three order component signal is synthesized, where two close orders, 4 and 4.2 , are designated. Among these three orders of the synthetic signal, their RMS amplitudes are a fixed value of 2, linearly increasing from 0 to 10 and from 2 to 12 for order 1, 4, and 4.2, respectively. The signal is designed to validate the performance of the online real-time monitoring system based on the proposed scheme in separating close orders that arise from multifunction transmission elements of a rotating system. Two close orders are characterized by the rpm-spectrogram, as shown in Fig. 7. The waveforms of order 4 and 4.2 were extracted by the scheme (Fig. 8); again, during the low speed phase below $800 \mathrm{rpm}$, these two close orders are mixing so that it causes the oscillation of computed RMS amplitudes. Figure 9 presents the error percentages, $e(n)$,

$$
e(n)=\frac{\left|\sqrt{\frac{1}{M} \sum_{k=(n-1) M+1}^{n M} y^{2}(k)}-\sqrt{\frac{1}{M} \sum_{k=(n-1) M+1}^{n M} x^{2}(k)}\right|}{\sqrt{\frac{1}{M} \sum_{k=(n-1) M+1}^{n M} x^{2}(k)}} \times 100 \%, \quad n=1,2,3, \ldots, N
$$

where $x(n)$ and $y(n)$ are respectively the designated and extracted waveforms for the evaluated waveforms, and the multiplication $M N$ is the data number for both $x(n)$ and $y(n)$. At low speed, below $800 \mathrm{rpm}$, the percentage of errors are larger due to the mixing amplitudes as shown in the Fig. 9. Then it is seen the error percentages for order 4 and 4.2 become smaller than $5 \%$ and $3 \%$ as the revolution speed increases. 


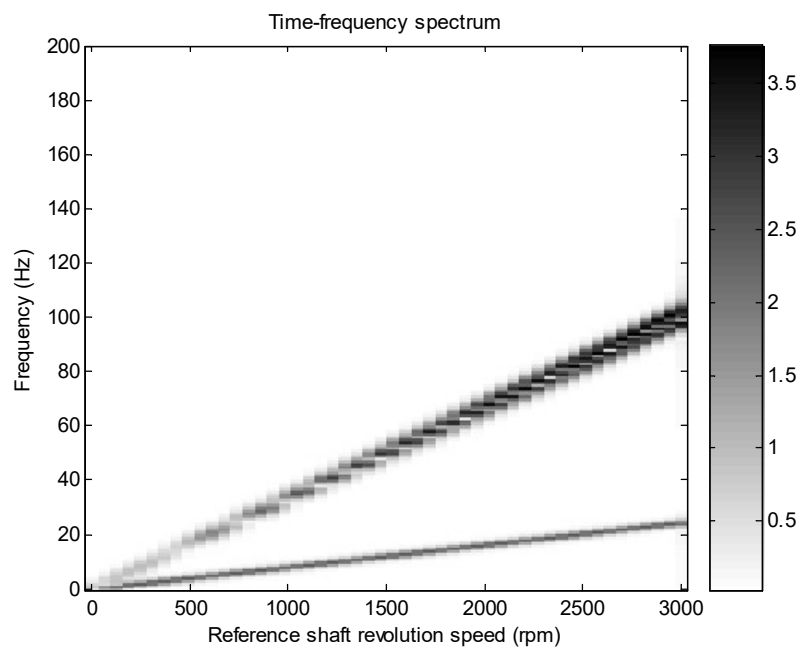

Fig. 7. rpm-spectrogram of synthetic signal 2 to characterize close orders (order 4 and 4.2).
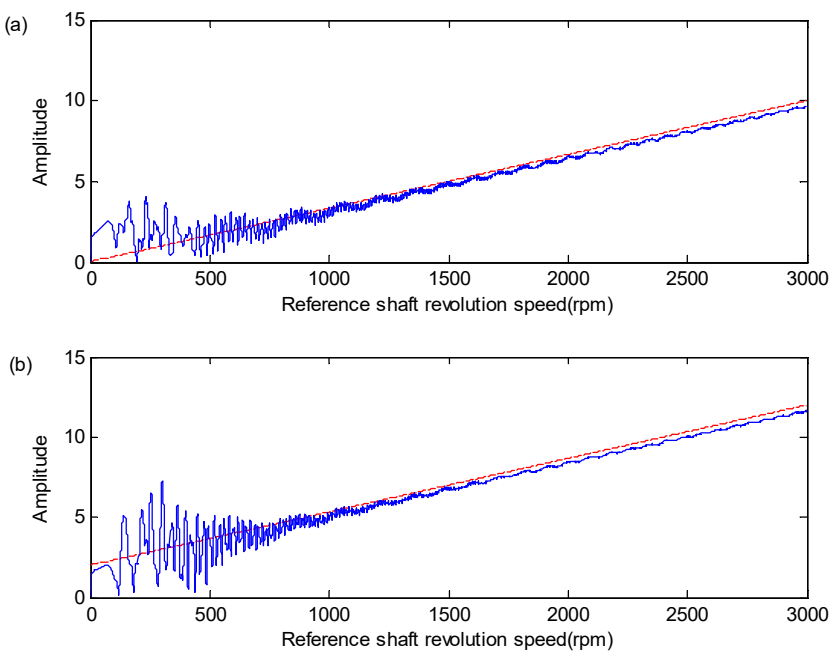

Fig. 8. Extracted (a) order 4 and (b) order 4.2 waveform for synthetic signal 2.
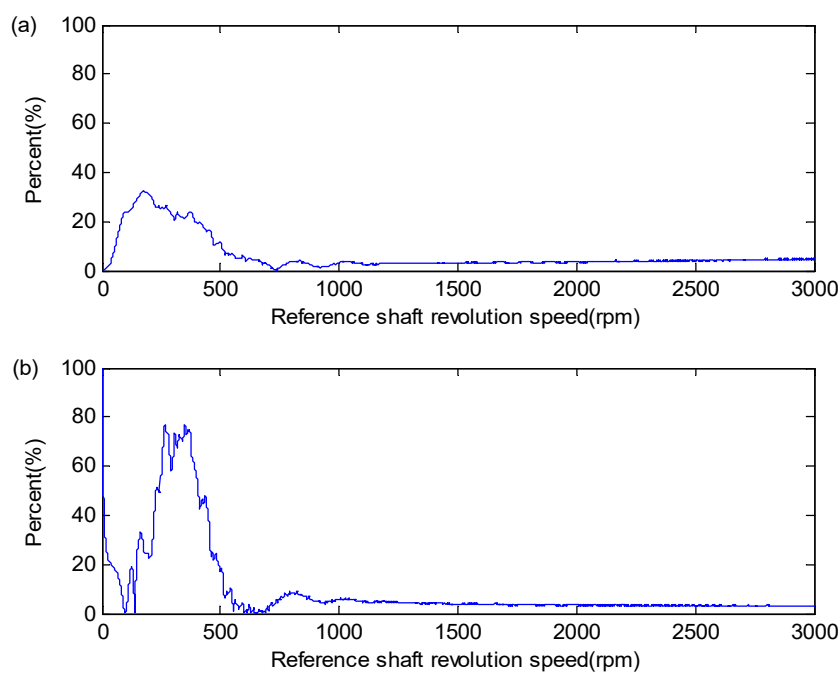

Fig. 9. Error percentages for evaluated (a) order-4 and (b) order-4.2 components. 


\subsection{Experimentation}

As mentioned in section 3, the rotor test bench with journal bearing and the multifunction transmission-element test bench were constructed to justify our developed on-line real-time monitoring system with the AV2KF_OT scheme that was coded in an embedded DSP. The increasing speed of the journal bearing system causes the fluid induced instability, thereby the test was for whether the online adaptive angular-velocity VKF order tracking can accurately determine the operating condition of the machine and then handle the system. As to the multifunction transmissionelement system, the use of proposed scheme was for promptly tracking specific orders from a multiple-close-order signal.

\subsubsection{Journal bearing test bench}

In this case study through the journal bearing rotor system, the fluid-induced instability with various whirl situations was designated to be detected through using two vertically-mounted Eddy current proximity probes. The test bench operates to speed up continuously to $7000 \mathrm{rpm}$ in $180 \mathrm{~s}$. The data of journal oscillation were acquired with a sampling frequency $10 \mathrm{kHz}$ and processed in a realtime manner using the one-step prediction scheme. As the machine speed gradually rises, the fluidinduced instability occurs. When these phenomena were generated by the rotor system, the online real-time monitoring system that is able to track specific vibration orders can immediately determine the machine running state. Figure 10 characterizes the incipient and on-going whirl instability; especially Fig. 10(b) shows the rpm spectrogram of extracted order- 0.47 component through the computation using the adaptive OT. The extracted orders clearly show that the whirl starts at 3200 rpm and ends around $6800 \mathrm{rpm}$, whereas the whip occurs with a frequency of $52 \mathrm{~Hz}$. The RMS amplitudes can be evaluated to indicate the occurrence of fluid-induced instability, and used for machine condition monitoring, as shown in Fig. 11. For further investigation on varied status of test bench through tuning the center-line adjuster to raise the speed of whirl occurrence, the developed monitoring system can still monitor the machine condition effectively. Likewise, Figs. 12 and 13 illustrate that the real-time monitoring system detects the incipient instability of the bench that postpones to a higher speed, $3300 \mathrm{rpm}$.
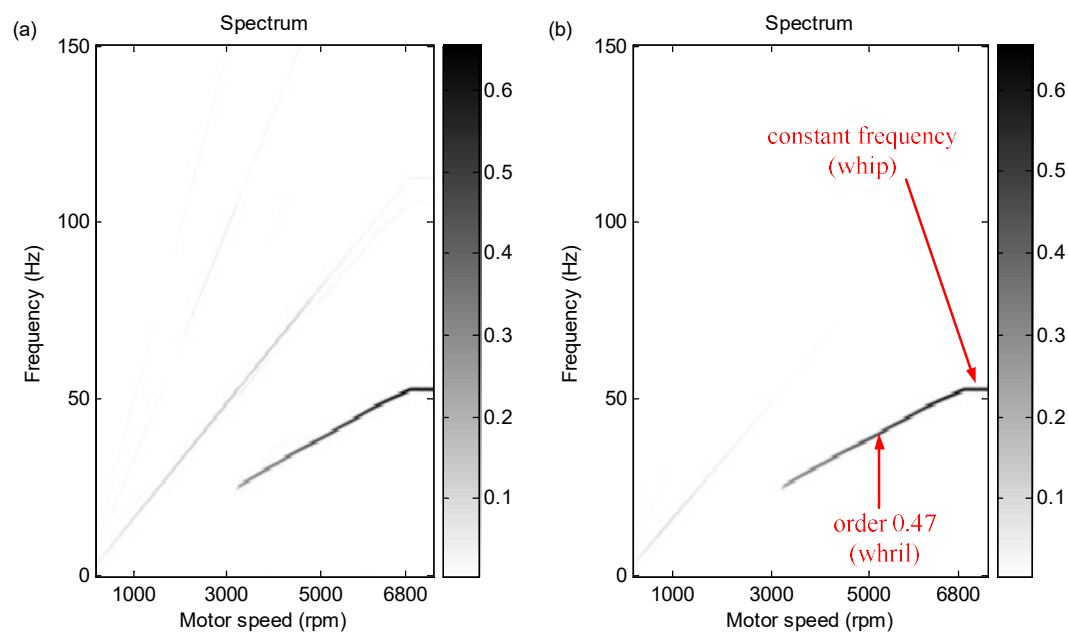

Fig. 10. rpm spectrograms of (a) measured vibration signal and (b) extracted order 0.47 component. 


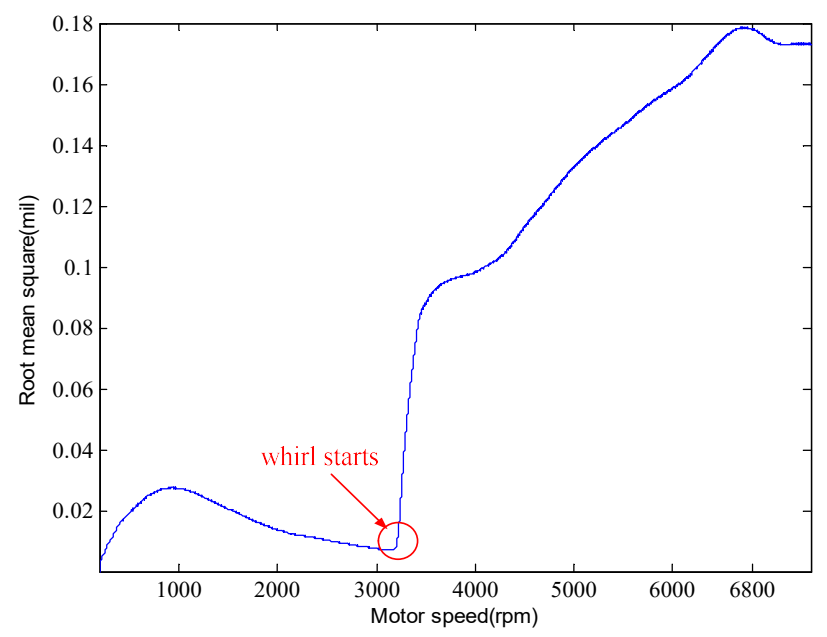

Fig. 11. Evaluated RMS of order 0.47 component along motor speed.
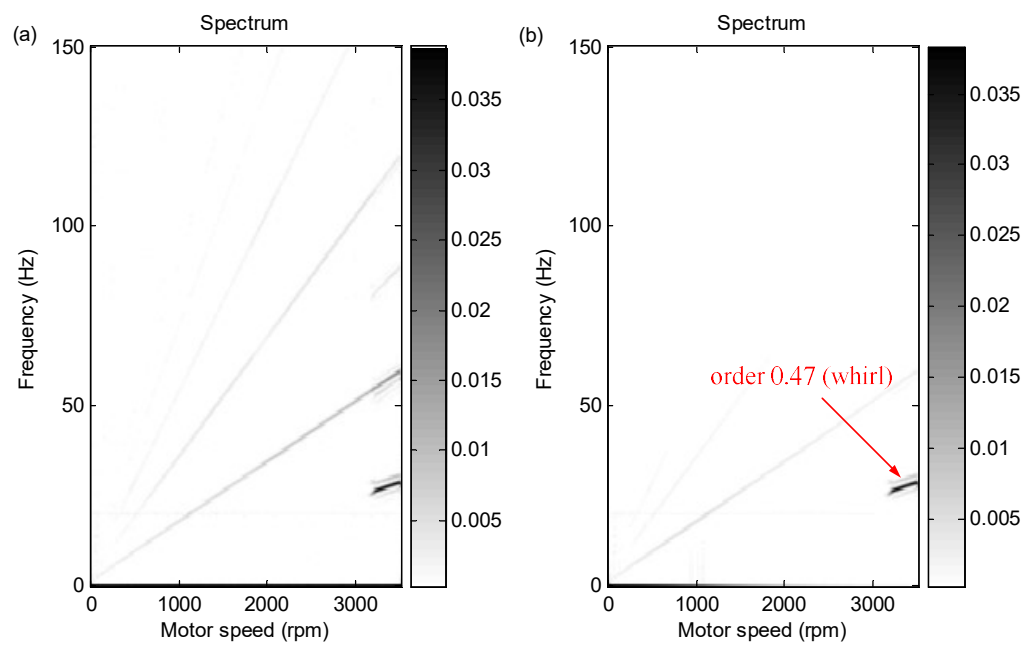

Fig. 12. rpm spectrograms of (a) measured vibration signal and (b) extracted order 0.47 component (after tuning the center-line adjuster).

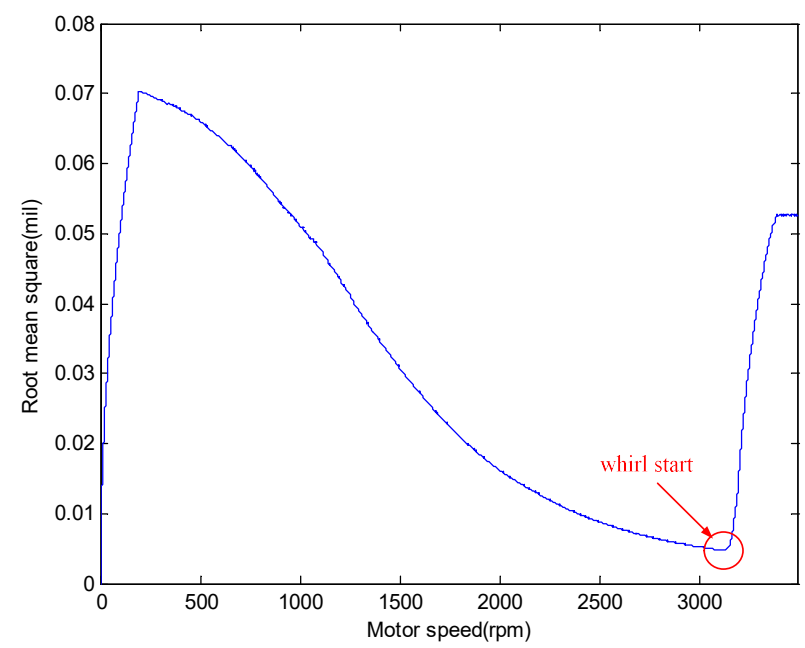

Fig. 13. Evaluated RMS of order 0.47 component along motor speed (after tuning the center-line adjuster). 


\subsubsection{Multifunction transmission-element test bench}

For the second case study using the transmission-element rotating system, the vibration of two close-order signatures was generated; that often occurs in a rotary machine with multiple transmission elements. The vibration signal with two close orders (order 1 and 1.31) arising from a slightly unbalanced shaft and the four times of belt speed was decoupled. The belt-speed frequency, fpulley, can be calculated as

$$
f_{\text {belt }}=\frac{d_{\text {pulley }} \times \pi}{l_{\text {belt }}} \times f_{\text {pulley }} \text {, }
$$

where $l_{\text {belt }}$ is the length of the belt, $d_{\text {pulley }}$ and $f_{\text {pulley }}$ denote the diameter and speed frequency of the driven pulley, respectively. It is noted that in the test the speed ratio of the belt driven set was 2.2. Replacing the values of $l_{\text {belt }}, d_{\text {pulley }}$ and $f_{\text {pulley }}(680 \mathrm{~mm}, 155 \mathrm{~mm}$, and $0.46 \mathrm{X})$ into Eq. (27) leads to $\mathrm{f}_{\text {belt }}=0.33$ $X$; here $X$ is the main-shaft speed (the same as the motor speed). As a result, the four times of the belt speed frequency is $1.32 \mathrm{X}$. The experiment was performed with the motor speed increasing from stationary to $3000 \mathrm{rpm}$ in $5 \mathrm{~s}$, and the sampling frequency of $5000 \mathrm{~Hz}$. An accelerometer (B\&K 4502) with a sensitivity of $8.83 \mathrm{mv} / \mathrm{g}$ was mounted above the gear box to measure the radial vibration. During the operation, an Odem flexible coupling which is used to transmit power causes a slightly unbalanced for the rotor with vibration signal of order 1 component. The speed ratio of belt-driven set is intentionally tuned to give 0.46 times of the belt speed frequency. Figure 14 (a) and (b) show the rpm spectrograms for both the original vibration signal and order-1 filtered out one; likewise, Fig. 15 (b) characterizes the signal with order 1.32 signature being filtered out through using the proposed method. This case example illustrates that the online real-time VK2-OT scheme with order/spectral tracking capacity can effectively monitor the operation status of a rotary mechanical system which generates close-order vibration signals.
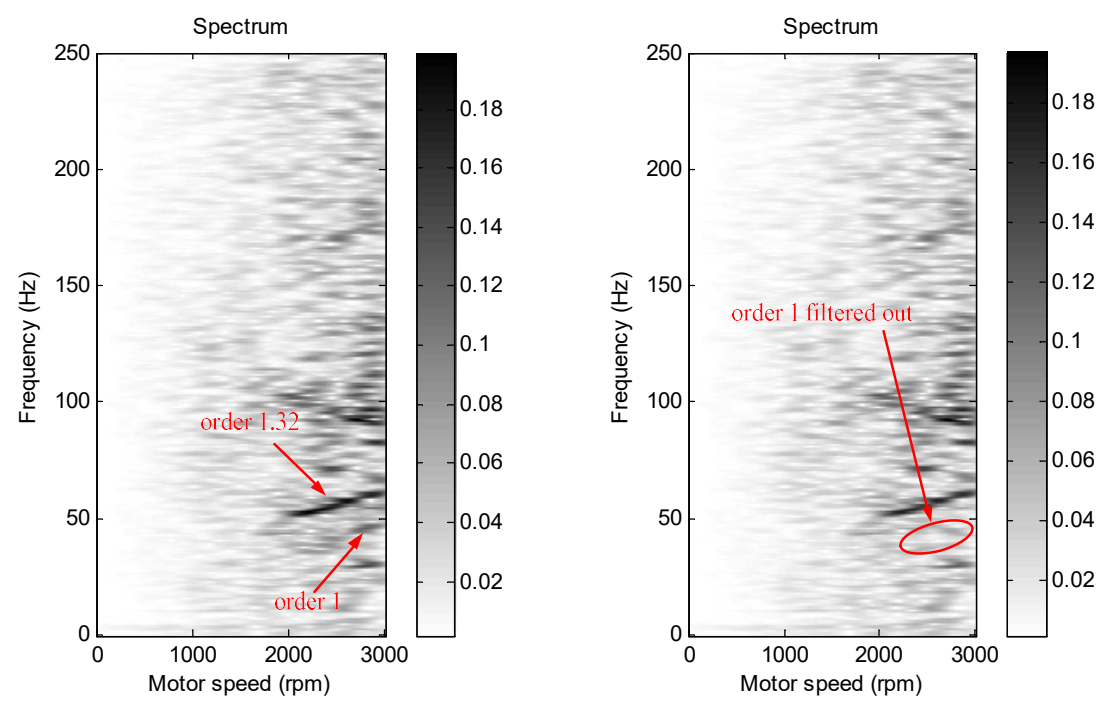

Fig. 14. rpm spectrograms of (a) the original signal and (b) the order-1 filtered out signal. 

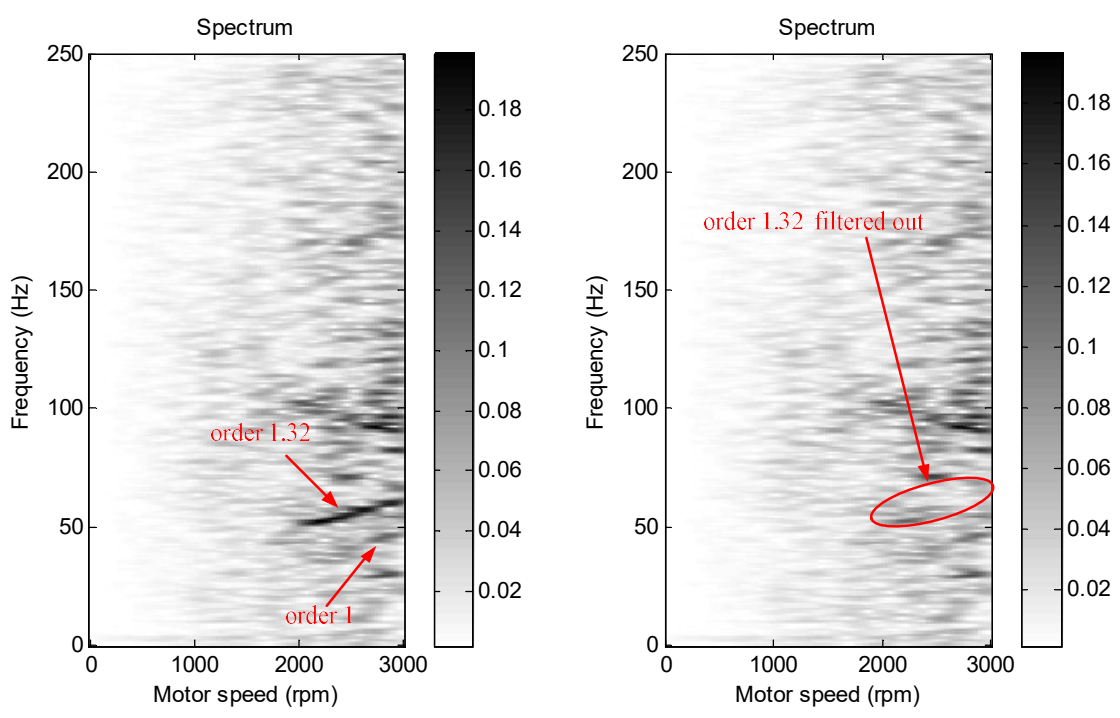

Fig. 15. rpm spectrograms of (a) the original signal and (b) the order-1.32 filtered out signal.

\section{Conclusion}

The paper proposed and implemented an online real-time monitoring system through using the adaptive angular-velocity VKF order tracking scheme coded through LabVIEW® and DSP chip module. First, two set of signals were synthesized to confirm its feasibility, and then the monitoring system was verified by two experimental test rigs, the journal-bearing rotor test bench and the multifunction transmission-element test bench, to understand its performance from the measured signals.

-- For the case on journal bearing rotor system, the monitoring of various fluid-induced instabilities, whirl (order 0.47) and whip (resonance), was explored. Further, through tuning the center-line adjuster the occurrence of whirl can be regulated to a varied preferred speed. The developed monitoring system can be employed to detect incipient whirl conditions.

-- For the case on multifunction transmission-element test bench, the monitoring system accurately separates two close orders, order 1 from unbalance and order 1.32 from four times of belt speed, from the measured vibration signals.

Acknowledgments: The authors thank to partially financial support by the Ministry of Science and Technology in Taiwan through grant MOST 107-2622-E-008-011-CC2.

Author Contributions: Ting-Chi Yeh performed the experiments and helped analyzing the data; Min-Chun Pan proposed the analysis methods, contributed materials/analysis tools, and wrote and revised the paper.

Conflicts of Interest: The authors declare no conflict of interest.

\section{References}

[1] M.-C. Pan, S.-W. Liao, and C.-C. Chiu, "Improvement on Gabor order tracking and objective comparison with Vold-Kalman filtering order tracking," Mechanical Systems and Signal Processing, vol. 21, pp. 653-667, 2007.

[2] P. R., "A new order tracking method for rotating machinery," Sound and Vibration, pp. 30-34, 1990.

[3] S. Qian, "Gabor expansion for order tracking," Sound and Vibration, vol. 37 (6), pp. 18-22, 2003.

[4] M.-C. Pan and J.-X. Chen, "Transmission noise identification using two-dimensional dynamic signal analysis," Journal of Sound and Vibration, vol. 262, pp. 117-140, 2003.

[5] H. Vold and J. Leuridan, "High Resolution Order Tracking at Extreme Slew Rates Using Kalman Tracking Filters," Shock and Vibration, vol. 2, 1993.

[6] H. Vold, Mains, M., and Blough, J., "Theoretical Foundations for High Performance Order Tracking with the Vold-Kalman Tracking Filter," SAE Technical Paper, 1997. 
[7] H. Vold, "Multi axle order tracking with the Vold-Kalman tracking filter," Sound and Vibration, vol. 31, No.5, pp. 30-34, 1997.

[8] M. C. Pan and Y. F. Lin, "Further exploration of Vold-Kalman-filtering order tracking with shaft-speed information-I: Theoretical part, numerical implementation and parameter investigations," Mechanical Systems and Signal Processing, vol. 20, pp. 1134-1154, 2006.

[9] M. C. Pan and Y. F. Lin, "Further exploration of Vold-Kalman-filtering order tracking with shaft-speed information-II: Engineering applications," Mechanical Systems and Signal Processing, vol. 20, pp. 1410$1428,2006$.

[10] M. R. Bai, J. Jeng, and C. Chen, "Adaptive Order Tracking Technique Using Recursive Least-Square Algorithm," Journal of Vibration and Acoustics, vol. 124, pp. 502-511, 2002.

[11] J.-D. Wu, C.-W. Huang, and R. Huang, "An application of a recursive Kalman filtering algorithm in rotating machinery fault diagnosis," NDT \& E International, vol. 37, pp. 411-419, 2004.

[12] M. F. Albright, Sh. Qian, "A Comparison of the Newly Proposed Gabor Order Tracking Technique vs. Other Order Tracking Methods," SAE Technical Paper, vol. 2001-01-1471, 2001.

[13] M.-C. Pan and C.-C. Chiu, "Investigation on improved Gabor order tracking technique and its applications," Journal of Sound and Vibration, vol. 295, pp. 810-826, 2006.

[14] S. Haykin, Adaptive Filter Theory. Upper Saddle River,NJ: Prentice Hall, 2002.

[15] M.-C. Pan and C.-X. Wu, "Adaptive Vold-Kalman filtering order tracking," Mechanical Systems and Signal Processing, vol. 21, pp. 2957-2969, 2007.

[16] M. Pan and W. Chu, "Adaptive Angular-Velocity Vold-Kalman Order Tracking Used in Characterizing Dynamic Signals Measured From Rotary Machines," in Proceedings of ASME IDETC/CIE 2009, San Diego, California, USA, 2009, pp. 153-158.

[17] M.-Ch. Pan*, W.-Ch. Chu and Duc-Do Le, 2016, “Adaptive Angular-Velocity Vold-Kalman Filter Order Tracking- Theoretical Basis, Numerical Implementation and Parameter Investigation," Mechanical Systems and Signal Processing, Vol. 81, pp. 148-161.

[18] Y. Guo and K. K. Tan, "Order-crossing removal in Gabor order tracking by independent component analysis," Journal of Sound and Vibration, vol. 325, pp. 471-488, 2009.

[19] Y. Guo and K.K. Tan, "High efficient crossing-order decoupling in Vold-Kalman filtering order tracking based on independent component analysis," Mechanical Systems and Signal Processing, vol. 24, pp. 1756$1766,2010$.

[20] K. S. Wang and P. S. Heyns, "Application of computed order tracking, Vold-Kalman filtering and EMD in rotating machine vibration," Mechanical Systems and Signal Processing, vol. 25, pp. 416-430, 2011.

[21] J.-L. Dion, C. Stephan, G. Chevallier, and H. Festjens, "Tracking and removing modulated sinusoidal components: A solution based on the kurtosis and the Extended Kalman Filter," Mechanical Systems and Signal Processing, vol. 38, pp. 428-439, 2013.

[22] D. E. Bently and C. T. Hatch, Fundamentals of rotating machinery diagnostics: Bently Pressurized Bearing Company, 2002.

[23] A. Muszynska, Rotordynamics: CRC Taylor \& Francis Group, 2005. 